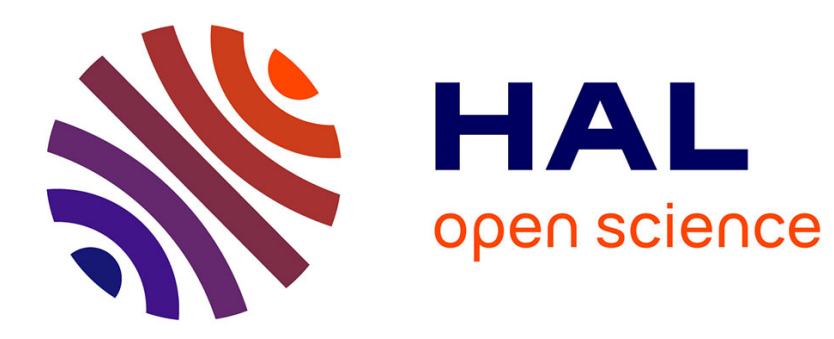

\title{
Optima on Hierarchies of Partitions
}

\author{
Jean Serra, Bangalore Ravi Kiran
}

\section{To cite this version:}

Jean Serra, Bangalore Ravi Kiran. Optima on Hierarchies of Partitions. 11th International Symposium on Mathematical Morphology and Its Applications to Signal and Image Processing (ISMM 2013), May 2013, Uppsala, Sweden. pp.147-158, 10.1007/978-3-642-38294-9_13 . hal-00802457

\section{HAL Id: hal-00802457 https://hal.science/hal-00802457}

Submitted on 19 Mar 2013

HAL is a multi-disciplinary open access archive for the deposit and dissemination of scientific research documents, whether they are published or not. The documents may come from teaching and research institutions in France or abroad, or from public or private research centers.
L'archive ouverte pluridisciplinaire HAL, est destinée au dépôt et à la diffusion de documents scientifiques de niveau recherche, publiés ou non, émanant des établissements d'enseignement et de recherche français ou étrangers, des laboratoires publics ou privés. 


\title{
Optima on Hierarchies of Partitions
}

\author{
Jean Serra and B. Ravi Kiran \\ Université Paris-Est, Laboratoire d'Informatique Gaspard-Monge, A3SI, ESIEE \\ $\{j$. serra, kiranr,\} @esiee.fr
}

\begin{abstract}
A new approach is proposed for finding optimal cuts in hierarchies of partitions by energy minimization. It rests on the notion of $h$-increasingness, allows to find best(optimal) cuts in one pass, and to obtain nice "climbing" scale space operators. The ways to construct $h$-increasing energies, and to combine them are studied, and illustrated by two examples on color and on textures.
\end{abstract}

\section{Introduction}

A hierarchy, or pyramid, of image segmentations is as a series of progressive simplified versions of an initial image, which result in increasing partitions of the space. We propose to reduce such pyramids to some best or optimal segmentation ${ }^{1}$. We shall not focus on the methods for obtaining the pyramids, and consider rather the whole hierarchies as starting points. Now, a multi-scale image description can rarely be considered as an end in itself. It often requires to be completed by some energy function $\omega$ that allows us to formalize optima. More precisely, three pieces of information interact, namely a pyramid $H$ of partitions of the space $E$ (possibly segmentations of an input image), some energetic function $f$ on $E$ which may be the initial image, or another one, and an energy $\omega$ over the partial partitions $\mathcal{D}(E)$, and which depends of $f$. Three questions arise then, namely:

1. Given a hierarchy $H$ of partitions and a energy $\omega$, how to obtain a new partition that minimizes $\omega$, without getting bogged down in the combinatorial complexity?

2. How a family $\left\{\omega^{j}, j \in J\right\}$ can be a scale space operator, and generate a sequence of optimal partitions that increase with $j$ ?

3. Most of the segmentations involve several features (colour, shape, size, etc.), that one can handle with different energies $\omega$. How to combine them, according to which grammar?

These questions, which are treated below in this order, have been taken up by several authors, for many years, and by various methods. Some of them [10], [6] simplify the combinatorial complexity by assuming that the energy of a partition

1 This work received funding from the Agence Nationale de la Recherche through contract ANR-2010-BLAN-0205-03 KIDIKO. 


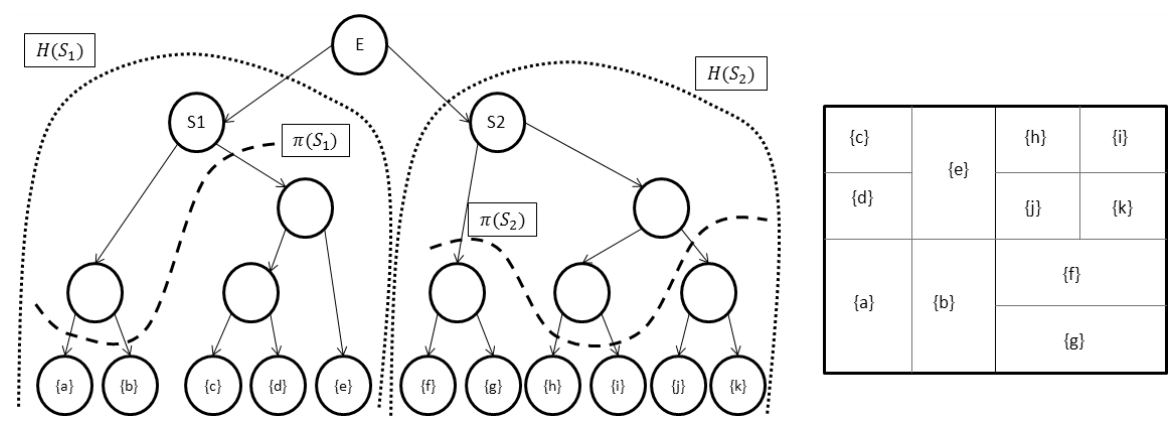

Fig. 1. Example of a dendrogram, with the corresponding partitions.

equals the sum of the energies of its classes, which permits the treat the above questions 1) and 3). However, one can wonder whether additivity is the very underlying cause, since alternative approaches [12] replace the sums by suprema and obtain similar properties. In fact, the corner stone for a method which aims to solve questions 1 ) to 3 ) is the notion of $h$-increasingness introduced in [11], which encompasses all above particular assumptions. This following sections demonstrate this.

\section{Hierarchies and cuts (reminder)}

\subsection{Hierarchies of partial partitions}

We denote by $E$ a $2-D$ topological space, such as a subset of $\mathbb{R}^{2}$ or $\mathbb{Z}^{2}$. A partition $\pi(S)$ associated with a set $S \in \mathcal{P}(E)$ is called partial partition of $E$ of support $S$ [9]. The partial partition of $S$ in the single class $S$ is denoted by $\{S\}$. The family of all partial partitions of set $E$ is denoted by $\mathcal{D}(E)$, or simply by $\mathcal{D}$. A hierarchy $H$ is a chain of partitions $\pi_{i}$, i.e.

$$
H=\left\{\pi_{i}, 0 \leq i \leq n \mid i \leq k \leq n \Rightarrow \pi_{i} \leq \pi_{k}\right\}
$$

where $\pi_{n}$ is the partition $\{E\}$ of $E$ in a single class, called the root. The classes of the finest partition $\pi_{0}$ are called the leaves. The intermediary classes are also called nodes. The number of leaves is supposed to be finite, so that $n$, the number of levels is also finite.

Let $S_{i}(x)$ be the class of partition $\pi_{i}$ of $H$ at point $x \in E$. Expression (1) means that at each point $x \in E$ the family $\left\{S_{i}(x), x \in E, 0 \leq i \leq n\right\}$ of those classes $S_{i}(x)$ that contain $x$ forms a finite chain of nested elements from the leaf $S_{0}(x)$ to $E$.

According to a classical result, a family $\left\{S_{i}(x), x \in E, 0 \leq i \leq n\right\}$ of indexed sets generates the classes of a hierarchy iff $i \leq j$ and $x, y \in \bar{E}$ implies

$$
S_{i}(x) \subseteq S_{j}(y) \text { or } S_{i}(x) \supseteq S_{j}(y) \text { or } S_{i}(x) \cap S_{j}(y)=\varnothing .
$$




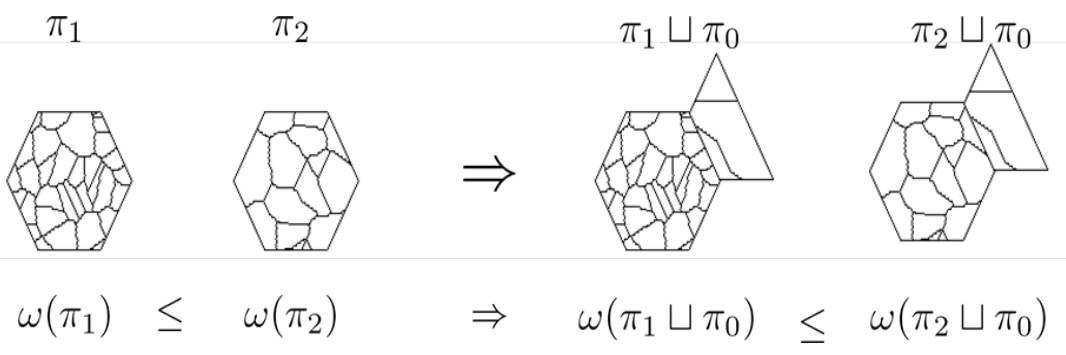

Fig. 2. Hierachical increasingness.

The partitions of a hierarchy may be represented by their classes, or by the saliency map of the edges, or again by a dendrogram where each node of bifurcation is a class $S$, as depicted in Figure 1. The classes of $\pi_{i-1}$ at level $i-1$ which are included in class $S_{i}(x)$ are said to be the sons of $S_{i}(x)$. Denote by $\mathcal{S}(H)$ the set of all classes $S$ of all partitions involved in $H$. Clearly, the descendants of each $S$ form in turn a hierarchy $H(S)$ of root $S$, which is included in the complete hierarchy $H=H(E)$.

\subsection{Cuts in a hierarchy}

Any partition $\pi$ of $E$ whose classes are taken in $\mathcal{S}$ defines a cut $\pi$ in a hierarchy $H$. The set of all cuts of $E$ is denoted by $\Pi(E)=\Pi$. Every "horizontal" section $\pi_{i}(H)$ at level $i$ is obviously a cut, but several levels can cooperate in a same cut, such as $\pi\left(S_{1}\right)$ and $\pi\left(S_{2}\right)$, drawn with thick dotted lines in Figure 1. Similarly, the partition $\pi\left(S_{1}\right) \sqcup \pi\left(S_{2}\right)$ generates a cut of $H(E)$. The symbol $\sqcup$ is used here for expressing that groups of classes are concatenated, i.e.

$$
S=S_{1} \sqcup S_{2} \Leftrightarrow S=S_{1} \cup S_{2} \text { and } S_{1} \cap S_{2}=\varnothing
$$

One can also define cuts inside any sub-hierarchy $H(S)$ of summit $S$, and similarly, $\Pi(S)$ stands for the family of all cuts of $H(S)$.

\section{Optimization and hierarchical increasingness}

\subsection{Energies and optimization}

The family of all p.p. of the leaves is denoted by $\mathcal{D}$. An energy on $\mathcal{D}$ is a non negative function $\omega: \mathcal{D} \rightarrow[0, \infty]$. In the following, $\mathcal{D}$ will be provided with several energies $\omega$, which may satisfy the two following axioms (for the existence of nice optimal cuts, and for their unicity):

i) $\omega$ is $h$-increasing, i.e.

$$
\omega\left(\pi_{1}\right) \leq \omega\left(\pi_{2}\right) \quad \Rightarrow \quad \omega\left(\pi_{1} \sqcup \pi_{0}\right) \leq \omega\left(\pi_{2} \sqcup \pi_{0}\right) .
$$


where $\pi_{1}$ and $\pi_{2}$ are two p.p. of same support $S$, and $\pi_{0}$ a p.p. of support $S_{0}$ disjoint of $S$ [11]. The geometrical meaning of Rel.(3) is depicted in Figure 2.

ii) $\omega$ is singular, when the energy $\omega(\{S\})$ of class $S$ is differs from that of any p.p. of $S$, i.e.

$$
\pi(S) \text { p.p. of }\{S\} \quad \Rightarrow \quad \omega(\{S\}) \neq \omega(\pi(S)) \text {. }
$$

The optimization problem involves three entities:

1. A pyramid $H$ of partitions of $E$ which segment an input image,

2. An energy $\omega$ on the family $\mathcal{D}(E)$ of all partial partitions of $E$,

3. An "energetic" function $f$ on $E$ which may be the initial image, or another one, which parametrizes energy $\omega$.

These three pieces of information are independent, and aim to determine the cuts that minimizes $\omega$, i.e. such that $\omega\left(\pi^{*}\right)=\inf \{\omega(\pi) \mid \pi \in \Pi(E)\}$. They are called below the optimal cuts.

\subsection{Optimal cut characterization}

Though the hierarchies are discrete, the number of their possible cuts explodes combinatorially: a small hierarchy of 200 leaves and 10 levels generates billions of cuts! How to find out the best one? By means of which vital lead? The $h$ increasingness (3) turns out to be too demanding and too general, since it does not take into account that we are dealing with hierarchies. We are thus lead to replace it by the following weaker but more adapted version of $h$-increasingness. We have to introduce the set $\mathcal{H}$ of all finite hierarchies of partitions of $E$.

Definition 1. An energy $\omega$ on $\mathcal{D}(E)$ is weakly $h$-increasing when for any hierarchy $H \in \mathcal{H}$, any disjoint nodes $S$ and $S_{0}$ of $H$, and any partition $\pi_{0}$ of $S_{0}$, we have

$$
\omega\left(\pi^{*}\right)=\inf \{\omega(\pi), \pi \in \Pi(S)\} \Rightarrow \omega\left(\pi^{*} \sqcup \pi_{0}\right) \leq \inf \left\{\omega\left(\pi \sqcup \pi_{0}\right), \pi \in \Pi(S)\right\}
$$

where $\Pi(S)$ stands for the finite set of all partitions of node $S$ involved in hierarchy $H$.

Clearly, $h$-increasingness implies weak $h$-increasingness, i.e. Rel.(3) $\Rightarrow$ Rel.(5). More precisely, Rel.(3) has been weakened just enough to obtain the theorem of optimal cut working in both senses. Indeed, we now have

Theorem 1. Let $H \in \mathcal{H}$ be a finite hierarchy, and $\omega$ an energy on $\mathcal{D}(E)$, and $S$ be a node of $H$ of sons $T_{1} . . T_{p}$. If $\pi_{1}^{*}, . . \pi_{p}^{*}$ are cuts of optimal energies of $T_{1} . . T_{p}$ respectively, then

$$
\pi_{1}^{*} \sqcup \pi_{2}^{*} . . \sqcup \pi_{p}^{*},
$$

is an optimal cut of $\Pi(S) \backslash\{S\}$, for any $H \in \mathcal{H}$ and any $T_{1} . . T_{p}$ in $H$, if and only if $\omega$ is weakly h-increasing. 
Proof for theorem 1 is given in [7].

When the $h$-increasing energy $\omega$ is also singular, then theorem 1 leads to the following key consequence

Corollary 1. Let $\omega$ be h-increasing and singular energy. Then for any $H \in \mathcal{H}$ and any node $S$ of $H$ with $p$ sons $T_{1} . . T_{p}$ of optimal cuts $\pi_{1}^{*}, . . \pi_{p}^{*}$, there exists a unique optimal cut of the sub-hierarchy of root $S$. It is either the cut $\pi_{1}^{*} \sqcup \pi_{2}^{*} . . \sqcup \pi_{p}^{*}$, or the one class partition $\{S\}$ itself:

$$
\omega\left(\pi^{*}(S)\right)=\min \left\{\omega(\{S\}), \omega\left(\pi_{1}^{*} \sqcup \pi_{2}^{*} . . \sqcup \pi_{p}^{*}\right)\right\}
$$

Corollary 1 is essential. It governs the choices of models for energies, and their implementations:

Firstly, the obtained optimal cut $\pi^{*}(E)$ is indeed globally less energetic than any other cut in $H$, but, moreover, each class $S \in \pi^{*}(E)$ is less energetic than any p.p. of $S$ into classes of $H$, and also less energetic than any p.p. composed of classes of $H$ and containing $S$. This is a strong property of regional minimum.

Secondly, the condition (3) of $h$-increasingness for an energy being a notion independent of any hierarchy, one can use a different $\omega$ for each of the $n$ levels of hierarchy $H$.

Thirdly, dealing with $h$-increasingness is sufficient. Fortunately so, because it is incomparably easier to check the $h$-increasingness of an energy than its possible weak $h$-increasingness.

Fourthly, the optimal cut coincides with the min-cut in the sense of the maxflow methods on graphs when one takes for source the set of leaves, and for sink the whole space $E$.

Finally one can always impose unicity, for example by taking systematically $\{S\}$ and not $\pi^{*}(S)$ in case of equal energies at node $S$. This technique of choice makes $h$-increasingness and sigularity compatible [7].

\subsection{Generation of $\boldsymbol{h}$-increasing energies}

An easy way to obtain a $h$-increasing energy consists in defining it, firstly, over all sets $S \in \mathcal{P}(E)$, considered as one class partial partitions $\{S\}$, and then in extending it to partial partitions by some law of composition. Then, the $h$ increasingness is introduced by the law of composition, and not by $\omega[\mathcal{P}(E)]$. The first two modes of composition which come to mind are, of course, addition and supremum, and indeed we can state

Proposition 1. Let $E$ be a set and $\omega: \mathcal{P}(E) \rightarrow \mathbb{R}^{+}$an arbitrary energy defined on $\mathcal{P}(E)$, and let $\pi \in \mathcal{D}(E)$ be a partial partition of classes $\left\{S_{i}, 1 \leq i \leq n\right\}$. Then the two extensions of $\omega$ to the partial partitions $\mathcal{D}(E)$ by addition and by supremum

$$
\omega(\pi)=\sum\left\{\omega\left(S_{i}\right), 1 \leq i \leq n\right\} \quad \text { and } \quad \omega(\pi)=\vee\left\{\omega\left(S_{i}\right), 1 \leq i \leq n\right\}
$$

are $h$-increasing energies. Moreover, if $\left\{\alpha_{j}, j \in J\right\}$ stands for a family of non negative weights, then the weighed sum $\sum \alpha_{j} \omega_{j}$ and supremum $\bigvee \alpha_{j} \omega_{j}$ of $h$ increasing energies turn out to be $h$-increasing. 
(Easy proof). A number of other laws are compatible with $h$-increasingness. One can also make $\omega$ depend on more than one class, on the proximity of the edges, on another hierarchy, etc..

\section{Scale increasingness and climbing energies}

The usual energies are often given by finite sequences $\left\{\omega^{\lambda}, \lambda \in \Lambda\right\}$ that depend on a real positive index, or parameter, $\lambda$ which takes $p$ different values, $p<\infty$. Therefore, the processing of hierarchy $H$ results in a sequence of $p$ optimal cuts $\pi^{\lambda *}$, of labels $\lambda \in \Lambda$. A priori, the $\pi^{\lambda *}$ are not ordered, but if they were, then we should obtain a nice progressive simplification of the optimal cuts. For getting it, we need to combine $h$-increasingness with the supplementary axiom (8) of scale increasingness, which results in the following climbing energies.

Definition 2. We call climbing energy any family $\left\{\omega^{\lambda}, \lambda \in \Lambda\right\}$ of energies over $\mathcal{D}$ which satisfies the three following axioms, valid for each $\omega^{\lambda}$ and for all $\pi \in \Pi(S), S \in \mathcal{S}$.

i) each $\omega^{\lambda}, \lambda \in \Lambda$, is $h$-increasing,

ii) each $\omega^{\lambda}, \lambda \in \Lambda$, is singular,

iii) the $\left\{\omega^{\lambda}, \lambda \in \Lambda\right\}$ are scale increasing, i.e. for $\lambda \leq \mu$, each support $S \in \mathcal{S}$ and each partition $\pi \in \Pi(S)$, we have

$$
\lambda \leq \mu \text { and } \omega^{\lambda}(S) \leq \omega^{\lambda}(\pi(S)) \Rightarrow \omega^{\mu}(S) \leq \omega^{\mu}(\pi(S)), \quad \pi \in \Pi(S), \quad S \in \mathcal{S} .
$$

Axiom i) compares the same energy at two different levels, whereas axiom iii) compares two different energies at the same level. The relation (8) means that, as $\lambda$ increases, the $\omega^{\lambda}$ preserve the sense of energetic differences between the nodes of hierarchy $H$ and their partial partitions. In particular when $\omega_{0}$ is $h$-increasing and singular, and when family $\left\{\omega^{\lambda}, \lambda \in \Lambda\right\}$ is climbing, then the two families $\{\lambda \omega\}$, and $\left\{\omega^{\lambda}+\omega_{0}, \lambda \in \Lambda\right\}$ are climbing.

\subsection{Ordering and computation of the optimal cuts:}

The climbing energies satisfy the very nice property to order the optimal cuts with respect to the parameter $\lambda[7]$ :

Theorem 2. Let $\left\{\omega^{\lambda}, \lambda \in \Lambda\right\}$ be a family of climbing energies, and let $\pi^{\lambda *}$ (resp. $\left.\pi^{\mu *}\right)$ denote the optimal cut of hierarchy $H$ according to the energy $\omega^{\lambda}\left(\right.$ resp. $\left.\omega^{\mu}\right)$. Then the family $\left\{\pi^{\lambda *}, \lambda \in \Lambda\right\}$ of the optimal cuts generates a hierarchy $H^{*}$ of partitions, i.e.

$$
\lambda \leq \mu \quad \Rightarrow \quad \pi^{\lambda *} \leq \pi^{\mu *}, \quad \lambda, \mu \in \Lambda .
$$

Computationally, the h-increasing condition (3) allows us to reach the optimal cut in one ascending pass, by the following Guigues'algorithm [6]:

- Scan in one pass all nodes of $H$ in ascending lexicographic order. 
- Determine at each node $S$ a temporary optimal cut of $H$ by comparing the energy of $S$ to that of the concatenation of the temporary optimal cuts of the (already scanned) sons $T_{k}$ of $S$.

In addition, the scale increasingness allows us to obtain the whole family of the optimal cuts in one ascending pass followed by a descending one [7] [6].

We will now review two families of climbing energies. The first one focuses on the additivity of the classes and the second on their supremum.

\section{$5 \quad$ Additive energies}

The additive $h$-increasing mode was introduced and studied by L. Guigues et Al. under the name of separable energies [6], for partitions with connected classes. For the aim of scale increasingness, the energy $\omega^{\lambda}(S)$ is written as a linear function of $\lambda$ :

$$
\omega^{\lambda}(S)=\omega_{\varphi}(S)+\lambda \omega_{\partial}(S) \quad S \in \mathcal{S} .
$$

The additive family $\left\{\omega_{\varphi}+\lambda \omega_{\partial}\right\}$ is climbing iff the term $\omega_{\partial}$ is sub-additive for union, i.e.

$$
\omega_{\partial}\left(\bigcup_{1 \leq u \leq q} T_{u}\right) \leq \sum_{1 \leq u \leq q} \omega_{\partial}\left(T_{u}\right)
$$

The climbing family $\left\{\omega^{\lambda}\right\}$ of Rel. (10) admits a nice lagragian interpretation if we view the term $\omega_{\partial}$ as a constraint on the functional $\omega_{\varphi}$ to minimize. According to Lagrange formalism, given one constraint $\omega_{\partial}$, the optimum is reached by means of a system of partial derivatives. Now remarkably the current approach replaces that by a unique climbing. As the term $\omega_{\partial}(\pi)$ decreases as $\lambda^{j}$ increases, we can climb the pyramid of the optimal cuts and stop (thus optimal $\lambda$ ) when the constraint is satisfied.

The most popular climbing additive energy was proposed by Mumford and Shah $[8]$ and evolved under various forms. Let $\pi(S)$ be the partition of a summit $S$ into its $q$ sons $\left\{T_{u}, 1 \leq u \leq q\right\}$ i.e. $\pi(S)=T_{1} \sqcup . . T_{u} . . \sqcup T_{q}$. The energy $\omega_{\varphi}$, called fidelity term, sums up the quadratic differences between $f$ and its average $m\left(T_{u}\right)$ in the various $T_{u}$, and the energy $\omega_{\partial}$, called regularity term weights by $\lambda^{j}$ the lengths $\partial T^{i}$ of the frontiers of all $T_{u}$, i.e.

$\omega^{j}(\pi(S))=\sum_{1 \leq u \leq q} \int_{x \in T^{u}}\left\|f(x)-m\left(T_{u}\right)\right\|^{2}+\lambda^{j} \sum_{1 \leq u \leq q}\left(\partial T_{u}\right)=\omega_{\varphi}(\pi)+\lambda^{j} \omega_{\partial}(\pi)$

where the weight $\lambda^{j}$ is a numerical increasing function of the level number $j$.

The two terms of Rel.(12) are far from being the only possible ones. The second example of Section 8 below brings textures into play via inter-class variances. In [7], the convexity of $S$ is introduced by comparing the positive and negative curvatures of $\partial S$. In [13], the quadratic differences of $\omega_{\varphi}$, in Rel.(12), are replaced by two intergrals of $f$ in the outer and inner parts of the dilate $\partial S \oplus B$ of $\partial S$ by a disc $B$, etc.. 


\section{Sup-generated energies}

Just as the sum-generated ones, the $\vee$-generated energies on the partial partitions are defined from an energy $\omega$ on $\mathcal{P}(E)$, followed by a law of composition, which is now the supremum

$$
\omega(\pi)=\omega\left(T_{1} \sqcup \ldots \sqcup T_{n}\right)=\vee\left\{\omega\left(T_{i}\right)\right\} .
$$

\subsection{Binary $\vee$-generated energies}

These energies are proposed by P.Soille [12], with several variants. For example, a numerical function $f$ is associated with the hierarchy $H$. The range of variation $\delta(S)=\max \{f(x), x \in S\}-\min \{f(x), x \in S\}$ of $f$ inside set $S$ defines the $h$ increasing binary energy $\omega^{k}(\langle S\rangle)=0$ when $\delta(S) \leq k$, and $\omega^{k}(\langle S\rangle)=1$ when not. The energy $\omega^{k}$ is extended to partial partitions by supremum, so that the class of the optimal cut at point $x \in E$ is the larger class of $H$ whose range of variation is $\leq j$. When the energy $\omega^{k}$ of a father equals that of its sons, one keeps the father when $\omega^{k}=0$, and the sons when not.

\subsection{Ordered energies}

When they are not binary, some $\vee$-generated energies are presented via an ordering condition. As previously, an energy is still associated with each subset $S$ of $E$. The axiom (3) of $h$-increasingness does not require we know the energy of all partial partitions. In particular, when the comparison of the partial partitions $\pi_{1}$ and $\pi_{2}$ reduces to that of their classes, then a law of composition becomes useless.

Definition 3. An energy $\omega$ on $\mathcal{D}$ is said to be ordered when for all pairs $\pi_{1}$, $\pi_{2} \in \mathcal{D}$ we have $\omega\left(\pi_{1}\right) \leq \omega\left(\pi_{2}\right)$ iff

-Both $\pi_{1}$ and $\pi_{2}$ admit the same support Supp,

-For all points $x \in S u p p$, for classes $S_{1}(x)$ and $S_{2}(x)$ in $\pi_{1}$ and $\pi_{2}$ respectively, the inequality $\omega\left[\left\{S_{1}(x)\right\}\right] \leq \omega\left[\left\{S_{2}(x)\right\}\right]$ holds.

An ordered energy $\omega$ is always $h$-increasing. When $S$ is the support of the partition $\pi=\sqcup T_{i}$, then $\omega(S) \leq \omega(\pi)$ iff $\omega(S) \leq \vee \omega\left(T_{i}\right)$, and we find again the $\checkmark$-composition.

Here is an example of ordered energy due to H.G.Akcay and S. Aksoy [1] who study airborne multi-band images and introduce (up to a small change) $\mu(S)=$ Area $(S) \times($ mean of all standard deviations of all bands in $S)$. They work with energy maximization. Allocate a non negative measure $\mu(S)$ to each node of a hierarchy $H$, where $\mu$ takes its values in a partially ordered set $M$, such as a color space. The energy $\omega$ is ordered by the two conditions

$$
\omega(S) \leq \omega\left(S^{\prime}\right) \Leftrightarrow S \supseteq S^{\prime} \text { and } \mu(S) \geq \mu\left(S^{\prime}\right) \quad S, S^{\prime} \in \mathcal{P}(E), \mu \in M .
$$




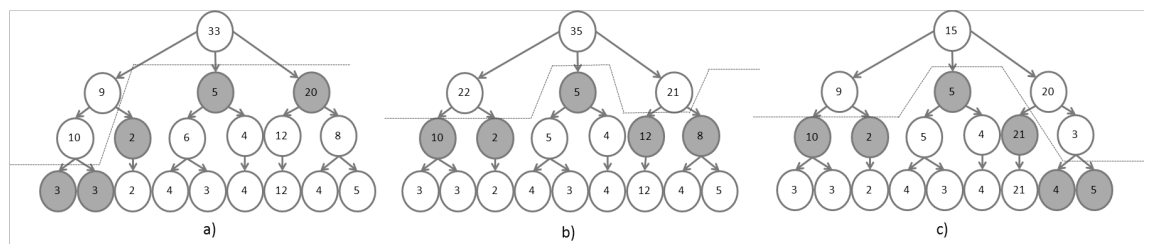

Fig. 3. Comparison of three laws of composition, a) by addition, b) by supremum, c) by ordering. The energies $\omega$ are indicated in the discs.

The node $S^{*}$ of the optimal cut at point $x$ is the highest more energetic than all its descendants. The best cut $\pi^{*}$ is obtained in one pass, by Guigues' algorithm [6].

Figure 6.2 summarizes the three major laws of composition. In a) the additive mode chooses the father $S$, when $\omega(S) \leq \sum \omega\left(T_{j}\right)$. In b) the mode by supremum chooses the $S$, when $\omega(S) \leq \vee \omega\left(T_{j}\right)$. Finally, in c) one takes the largest node which is more energetic than all its descendants(maximization of $\omega$ ).

\subsection{Composition of $\vee$-generated energies}

Though the weighted supremeum of $\vee$-generated energies is $h$-increasing (eqn.13), the infimum is not. In practice, this half-result is nevertheless useful, since the $\vee$, paradoxically, expresses the intersection of criteria. For example, when the function $f$ to optimize is colour, one can take for energies:

- $\omega_{1}(S)=0$ when $\delta \operatorname{Lum}(S)<k_{1}$, and $\omega_{1}(S)=1$ when not, - $\omega_{2}(S)=0$ when $\delta \operatorname{Sat}(S)<k_{2}$, and $\omega_{2}(S)=1$ when not.

Then the $h$-increasing energy $\omega_{1}(S) \vee \omega_{2}(S)=0$ when $S$ is constant enough for both luminance and saturation.

\section{$7 \quad$ Partial optimizations}

Covering the whole space with some optimal partition is not always an aim. Some studies require doing it, but in others ones the regions of interest are limited, and clearly marked out by the context. Moreover, the leave partition often includes a good many classes due to noise. And thirdly, the hierarchies generated by connected filters may comprise a large number of singleton classes. For example, Figure 4 b) and c) depict the flat zones obtained by an alternating filter by reconstruction acting on the 25098 image a). All black pixels indicate the singleton flat zones. When climbing the hierarchy, most of these point classes are covered by extended classes, which are more significant. Therefore we can just ignore the singletons when the classes of $H$ are given an energy.

In other situations, some classes may be considered as non relevant because they are too small, or too large, or too far from the zone of interest, or of a non wanted hue, etc...In all cases, they are clearly identified, so that some label 


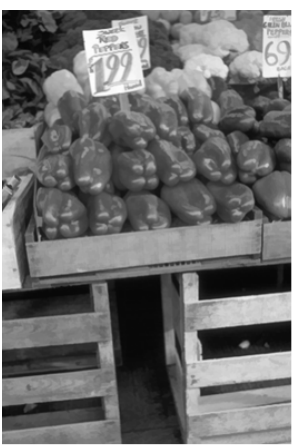

a)

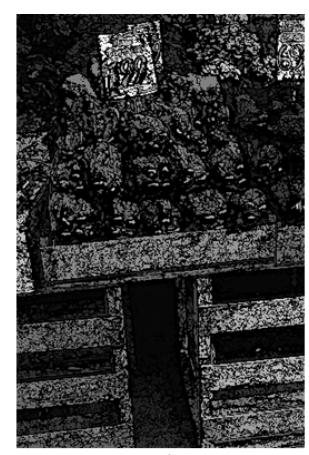

b)

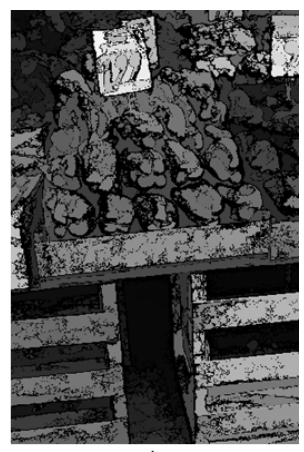

c)

Fig. 4. a) image 25098 from Berkeley database b) and c) alternating filters of a), of sizes 1 and 5 .

can indicate that they have not to intervene when computing the optimal cut. Denote by $\mathcal{W}(E) \subseteq \mathcal{P}(E)$ the set of all these undesirable classes. The energies $\omega$ must satisfy the condition that, for all families $\left\{S_{i}\right\} \subseteq \mathcal{P}(E)$ and all families $\left\{W_{j}\right\} \in \mathcal{W}(E)$ such that $\left(\cup_{i} S_{i}\right) \cap\left(\cup_{j} W_{j}\right)=\varnothing$, we have

$$
\omega\left(\left(\sqcup_{i} S_{i}\right) \sqcup\left(\sqcup_{j} W_{j}\right)\right)=\omega\left(\sqcup_{i} S_{i}\right) .
$$

The energy of the partial partition of classes $\left\{S_{i}\right\}$ must not change when outside $\left\{W_{j}\right\}$ classes are added. It means that $\omega(W)=0$ when the law of composition invoved in $\omega$ is the sum or the supremum, and that $\omega(W)=\infty$ when it is the infimum. When $\omega$ is $h$-increasing, the computation of the optimal cut is unchanged, but now results in a partition which may contain $W$ classes.

\section{Two examples}

We now develop two examples of additive energies which aim to show how the choice of the energies governs the extraction of specific features (color, textures). We start from the energy proposed in [10], and change it by adding new terms. A hierarchy $H$ of Uppsala ducks has been obtained by previous segmentations of the luminance $l=(r+g+b) / 3$ based on [5]. We want to find the best cut for a compression rate of 20 . In each class $S$ of $H$, the simplification consists in replacing the function $f$ by its colour mean (mean over all 3 channels) $\bar{l}(S)=\frac{\Sigma_{x \in S} I(x)}{\operatorname{card}(S)}$. In a first experiment the energy $\omega_{\text {lum }}(S)$ has for fidelity term $\omega_{\varphi}(S)$ the quadratic error, while the regularity term $\omega_{\partial}(S)$ is the coding cost of class $S$, by taking 2 bits for each frontier element, and 24 bits assigned to code $m(S)$ :

$$
\omega_{\text {lum }}(S)=\sum_{x \in S}\|l(x)-\bar{l}(S)\|^{2}+\lambda(24+|\partial S|)
$$




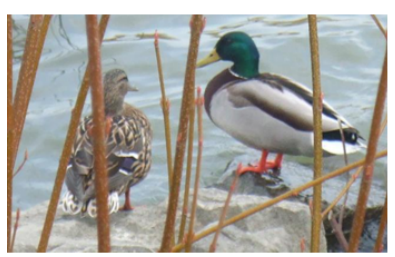

a)

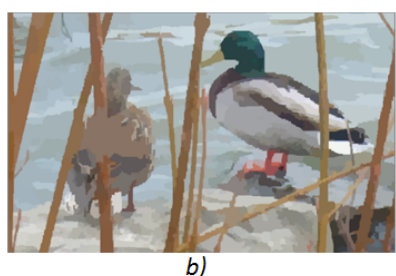

b)

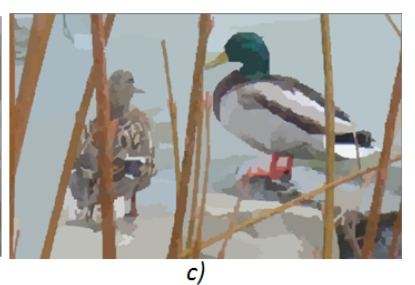

Fig. 5. a) Inititial Uppsala ducks; Optimal cuts b) by Luminance c) by Chrominance

The cost $\omega_{\partial}\left(\pi^{*}\right)$ of the best cut decreases as $\lambda^{j}$ increases, therefore we can climb the pyramid of the best cuts and stop when $\omega_{\partial}(\pi) \simeq$ pixels number $/ 20$. It results in Fig. 5b, where we see that the female duck is not nicely simplified. In the second experiment, we just replace the luminance $l$, in Rel.(15) by the chrominance $c$, i.e. by the projection of $(r, g, b)$ on the plane orthogonal to the main axis $(1,1,1)$. This simplifies the image while keeping partitions which minimize the variance of the chrominance vector $c$. The new optimal cut is depicted in Fig 5c. Both ducks are better separated from the foreground of the herbs, and from the background of the river.

$$
\omega_{\text {textures }}(S)=\omega_{\text {chrom }}(S)+\sum_{S \prime \in \text { sons }(S)} \frac{K}{\sigma^{2}\left(\operatorname{Area}\left(S^{\prime}\right)\right)}
$$

The second example addresses to the recognition of textures in the trees, in the walls and in the water of the Uppsala river of Fig. 6a. The new energy $\omega_{\text {textures }}$ of Rel.(16) keeps the first two terms of the previous experiment.

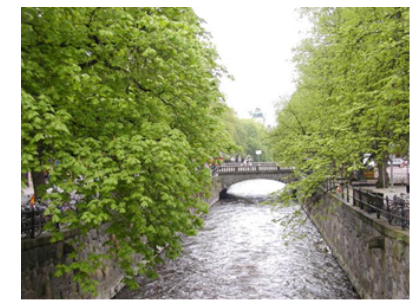

a)

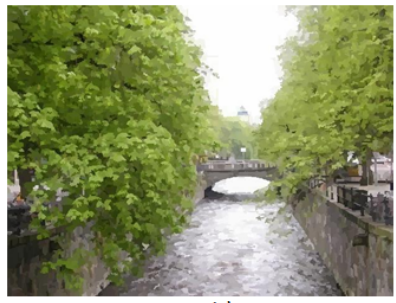

b)

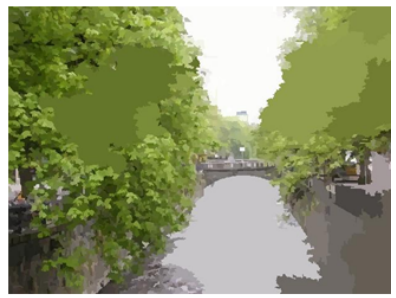

c)

Fig. 6. a) Uppsala river; b) and c) best cut of a) according to energy (16), for $\lambda=100$, and for $K=10^{12}$ (in b)), and $K=10^{14}$ (in c)).

The third term of (16) introduces the textures via the variances of the sons. It decreases more drastically when the areas of the sons of $S$ have similar sizes. Fig. $6 \mathrm{~b}$ and $\mathrm{c}$ depict the best cuts for two values of the parameters. Intuitively, texture 
features are formulated into this multi-scale framework where the optimal scale parameter combines the effect of chrominance and structure of texture into one global energy function, thus showing the flexibility of the framework.

\section{Conclusion}

The primary contributions of this theoretical paper were:

- Hierarchical optimizations based on $h$-increasingness, while also giving the conditions for general classes to be $h$-increasing.

- Defining non negative global climbing energies that allow to perform sequences of increasing optimizations over a hierarchy of segmentations.

- Demonstrating how to formulate multiple constraint functions over the image space in order to lead to different optimal segmentations. Two examples, one with colour image segmentation and one with texture enhancement were shown.

Acknowledgements We are thankful to Professor Fernand Meyer for his helpful comments on the converse of theorem 1.

\section{References}

1. Akcay, H.G., Aksoy, S.: Automatic detection of geospatial objects using multiple hierarchical segmentations. IEEE T. Geoscience and Remote Sensing 46 (2008) pp.2097-2111

2. Angulo J., Serra, J., Modeling and segmentation of colour images in polar representations Image and Vision Computing 25 (2007) 475-495.

3. Arbelez, P., Cohen L., Constrained Image Segmentation from Hierarchical Boundaries CVPR, (2008)

4. Cardelino, J., Caselles, V., Bertalmº, M., Randall, G.: A contrario hierarchical image segmentation. In: IEEE ICIP 2009, Cairo, Egypt (2009)

5. Cousty, J., Najman, L.: Incremental Algorithm for Hierarchical Minimum Spanning Forests and Saliency of Watershed Cuts. In: Soille, P., Pesaresi, M., Ouzounis, G.K.(eds.) ISMM 2011. LNCS, vol. 6671 Springer, Heidelberg (2011) 272-283

6. Guigues L., Cocquerez J.P., Le Men H., Scale-Sets Image Analysis, Int. Journal of Computer Vision 68(3), 289-317, 2006.

7. Kiran B.R., and Serra J., Global optimizations on hierarchies of segmentations(to appear in Pattern Recognition)

8. Mumford D. and Shah J., Boundary Detection by Minimizing Functionals, in Image Understanding, S. Ulmann and W. Richards Eds, 1988.

9. Ronse, C., Partial partitions, partial connections and connective segmentation. Journal of Mathematical Imaging and Vision 32 (2008) 97-125

10. Salembier P., Garrido L., Binary Partition Tree as an Efficient Representation for Image Processing, Segmentation, and Information Retrieval. IEEE Trans. on Image Processing, 2000, 9(4): 561-576.

11. Serra, J., Hierarchy and Optima, in Discrete Geometry for Computer Imagery, I. Debled-Renneson et al.(Eds) LNCS 6007, Springer 2011, pp 35-46

12. Soille, P., Constrained connectivity for hierarchical image partitioning and simplification. IEEE Transactions on PAMI 30 (2008)1132-1145

13. Xu Y., Géraud T., Najman L., Morphological Filtering in Shape Spaces: Applications using Tree-Based Image Representations. ICPR 2012 